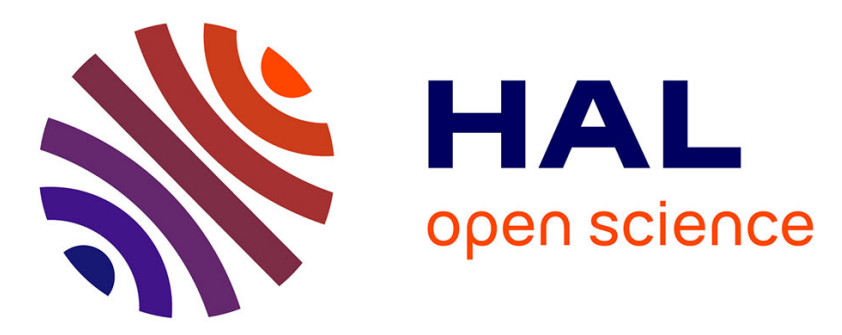

\title{
Au-Ni solid solutions studied by numerical relaxation
}

\author{
J. Eymery, F. Lançon, L. Billard
}

\section{To cite this version:}

J. Eymery, F. Lançon, L. Billard. Au-Ni solid solutions studied by numerical relaxation. Journal de Physique I, 1993, 3 (3), pp.787-802. 10.1051/jp1:1993163 . jpa-00246758

\section{HAL Id: jpa-00246758 https://hal.science/jpa-00246758}

Submitted on 1 Jan 1993

HAL is a multi-disciplinary open access archive for the deposit and dissemination of scientific research documents, whether they are published or not. The documents may come from teaching and research institutions in France or abroad, or from public or private research centers.
L'archive ouverte pluridisciplinaire HAL, est destinée au dépôt et à la diffusion de documents scientifiques de niveau recherche, publiés ou non, émanant des établissements d'enseignement et de recherche français ou étrangers, des laboratoires publics ou privés. 
Classification

Physics Abstracts

$61.55 \mathrm{H}-61.50 \mathrm{~L}$

\title{
Au-Ni solid solutions studied by numerical relaxation
}

\author{
J. Eymery, F. Lançon and L. Billard \\ CENG, Département de Recherche Fondamentale sur la Matière Condensée, Service des \\ Matériaux et Microstructures, B.P. 85X, 38041 Grenoble Cedex, France
}

(Received 20 July 1992, accepted in revised form 16 November 1992)

\begin{abstract}
Résumé. - Un potentiel semi-empirique, issu de la méthode des liaisons fortes dans l'approximation du second moment, est utilisé pour étudier la solution solide Au-Ni à $0 \mathrm{~K}$. L'énergie de formation, le désordre topologique et les constantes élastiques sont analysées pour toute l'étendue de concentration. Les résultats sont en très bon accord avec les données expérimentales disponibles et peuvent être interprétés par une frustration des liaisons atomiques par le réseau cfc. L'effet de taille est prépondérant devant l'effet chimique et l'analyse des sites interstitiels montre que l'environnement chimique local conduit à une levée de dégénérescence des volumes tétraédriques du réseau cfc.
\end{abstract}

\begin{abstract}
A semi-empirical potential based on the tight-binding second moment approximation is used to study Au-Ni solid solutions at $0 \mathrm{~K}$. The energy of formation, the topological disorder, and the elastic constants are analysed within the whole concentration range. The results coincide very well with available experiments and may be explained by a frustration of the interatomic bondings by the fcc lattice. The size effect predominates over the chemical one and the analysis of interstitial sites shows that the local chemical surroundings lead to a splitting of the fcc lattice tetrahedral volumes.
\end{abstract}

\section{Introduction.}

Computer simulations provide a powerful tool for studying the physical properties of solid solutions. First principle theory leads to the prediction of the energies of formation of binary compounds [1]. However, the mixing energy of the solid solution within the whole concentration range, especially when strong topological relaxation exists, is difficult to reproduce [2, 3]. Recently, elastic and chemical contributions were considered separately [4] to predict alloys energetics and the average lattice parameter in the framework of equivalentcrystal theory. But, within these methods, the local strains were not taken into account and topological microscopic properties (and their effects) could not be estimated. The use of interatomic potentials is appropriate for studying this problem and it allows one to extract statistical information from large systems.

Au-Ni alloys exhibit a miscibility gap in the solid state of the phase diagram which has stimulated a lot of thermodynamic and atomistic calculations $[5,6,7,8]$. A continuous 
substitutional solid solution is found at high temperature while an extensive region of separation into two fcc solid solutions occurs at temperatures below $1150 \mathrm{~K}$ [9]. The lattice distortion energy resulting from the strong size difference between unlike atoms (around $15 \%$ ) is responsible for the segregation behaviour $[5,6]$.

In this paper, we employ a simple semi-empirical potential based on the second moment approximation of the tight-binding description of metallic cohesion. With this potential, we analysed some properties at $\mathrm{OK}$ of the $\mathrm{Au}-\mathrm{Ni}$ metastable solid solution.

Firstly, it is shown that we obtain a reliable potential for the pure metals from fitting data (cohesion energy, equilibrium lattice parameter, monovacancy-formation energy) and from Rose's equation of state [10].

Secondly, by introducing interspecies interactions, we detail alloying energies (energy of formation of the solid solution and compounds, and heat of the solution in infinite dilution limit), topological properties (deviation from Vegard's law, partial length distributions and analysis of interstitial sites) and the elastic behaviours ( $c_{l j}$ and bulk modulus). Most of the results can be directly compared to the experiments and the influence of size effect on these various properties is clearly shown.

\section{Interatomic potential.}

2.1 GENERALITY. - The energy of a monoatomic metal in the second moment approximation is $[11,12]$ :

$$
E=\frac{1}{N} \sum_{1}^{N}\left[\sum_{j} A \exp \left\{-p\left(\frac{r_{l j}}{r_{0}}-1\right)\right\}-\left(\sum \xi^{2} \exp \left\{-2 q\left(\frac{r_{l j}}{r_{0}}-1\right)\right\}\right)^{1 / 2}\right]
$$

where $N$ is the number of atoms in the metal. The normalization of the length is made by $r_{0}$ which is a fixed parameter equal to the nearest-neighbour distance at $0 \mathrm{~K} . r_{i j}$ is the distance between the atoms $i$ and $j . \xi, q, A, p$ are the parameters to be determined. The summation over $j$ can be extended over several coordination shells $\left(r_{l j}<r_{\mathrm{c}}\right.$. cut-off radius). The exponential functions are truncated at a distance $r_{\mathrm{t}}$ and we interpolate both terms in relation (1) between $r_{\mathrm{t}}$ and $r_{\mathrm{c}}$ with polynomials of the fifth degree. This ensures continuity and derivability of the energy (at $r_{\mathrm{t}}$ and $r_{\mathrm{c}}$ ) up to the second order and allows to directly compute the second order elastic constants of pure components.

Most of the cohesive properties of transition metals are related to the second moment of the d-band density of states [11]. The second term of equation (1) corresponds classically to the width of this d-band.

The same formalism has been applied recently [13] to noble metals (with a full d-band) : the attractive term could result from the shift of the d-band. This shift is proportional to the square root of the second moment. The system is stabilized with a repulsive pairwise Born-Mayer interaction (first term of Eq. (1)).

This $N$-body potential does not have the drawbacks of pair potentials like the Cauchy relation : $\mathrm{C}_{12}=\mathrm{C}_{44}$ for cubic crystal. It has been used successfully to reproduce thermodynamical and structural properties of fcc transition metals [14] and to analyse the (110) surface stability in noble metals [13]. (Note that this model predicts the right inward relaxation of the surface plane contrary to pair interactions which lead to outward relaxations.) The same authors have tested the anharmonicity of the potential by inspecting dynamic properties in the first-neighbour approximation (frequency spectrum, thermal expansion and mean square displacement).

Equation (1) is also in accordance with other potentials used to simulate large systems of condensed matter physics, for instance, the Embedded Atom Method (EAM) [15, 16] where 
the summation of the band term is replaced by the local electron density and the square root is replaced by a many-body embedding function. The empirical Finnis and Sinclair (FS) approach $[17,18]$ is also very close to this work : a square-root embedding function has been chosen for both noble and transition metals, but the fitted coefficients are more numerous to adjust both the equilibrium lattice parameter, the cohesive energy, the three elastic constants of the fcc structure, a lower bound of the unrelaxed vacancy formation energy and the stacking fault energy.

2.2 FITTING PROCEDURE FOR PURE METALS. - The overlapping of the d-orbitals in transition metals [11] means that $\frac{p}{q}$ lies between 2 and 5. The direct adjustment, using the NewtonRaphson procedure, of $\xi, q, A, p$ to get $r_{0}, E_{0}$ (cohesion energy of the fcc structure), $B$ (bulk modulus) (See Tab. I for numerical inputs) and one elastic constant amongst $c_{11}, c_{12}$, $c_{44}$ or $c^{\prime}=\left(c_{11}-c_{12}\right) / 2$ leads to high Ni values of $\frac{p}{q}$ when the summation is extended beyond the first interaction shell [19]. Therefore, $q$ parameters are determined within reliable values found in the literature $[13,14]$.

The three remaining parameters are adjusted on the numerical values in table $I$ of quantities at $0 \mathrm{~K}$. We have verified that $r_{\mathrm{r}}=1.2 r_{0}$ is a correct starting point for the interpolation. The cutoff radius $r_{\mathrm{c}}$ is fixed so that the second derivative of the interpolation function has the fastest decrease as possible. This criterion is important in order to avoid any instabilities in the determination of elastic constants (particularly when the bond length distribution is broad). Table II gives the values of adjusted parameters.

Table I. - The three experimental quantities to be fitted: $r_{0}(\AA), E_{\mathrm{coh}}(\mathrm{eV})[42]$ and $B\left(10^{11} \mathrm{~Pa}\right)[43]$.

\begin{tabular}{|c|c|c|c|}
\hline & $r_{0}$ & $-E_{0}$ & $B$ \\
\hline $\mathrm{Au}$ & 2.880 & 3.78 & 1.810 \\
\hline $\mathrm{Ni}$ & 2.489 & 4.44 & 1.876 \\
\hline
\end{tabular}

Table II. - Values of the potential parameters for the repulsive and attractive terms of the pure metals. $A$ and $\xi$ are in $\mathrm{eV}, p$ and $q$ are dimensionless and the radii are normalized by the first-neighbour distances given in table $I$.

\begin{tabular}{|c|c|c|c|c|}
\hline Repulsive term & $A$ & $p$ & $r_{\mathrm{t}}$ & $r_{\mathrm{c}}$ \\
\hline $\begin{array}{c}\text { «AuAu » } \\
\text { « NiNi » }\end{array}$ & 0.2316 & 10.2053 & 1.2 & 1.5283 \\
& 0.1486 & 9.8858 & 1.2 & 1.5390 \\
\hline Attractive term & $\xi$ & $q$ & $r_{\mathrm{t}}$ & $r_{\mathrm{c}}$ \\
\hline $\begin{array}{c}\text { «AuAu » } \\
\text { « NiNi » }\end{array}$ & 1.8868 & 4.3098 & 1.2 & 1.5887 \\
\hline
\end{tabular}


To check the validity of our potential, we calculate the elastic constants and the vacancy formation energy. In a centrosymmetric lattice, the atomic displacements follow the macroscopic strain [20] and then elastic constants can be derived from equation (1). A simulation is performed to get the monovacancy-formation energy of the pure materials : a periodic cell of 3999 atoms and one vacancy is relaxed at zero pressure (relaxation details are given below). The calculated values are compared in table III with experimental results at $0 \mathrm{~K}$. The agreement is quantitatively good; the largest discrepancy is found for $c_{44}^{\mathrm{N} 1}$ although, $c_{44}^{\mathrm{Au}}$ is quite good. This behaviour certainly depends on the form of the potential which is too simple (for the bcc transition metals, the second moment approximation does not describe the density of states sufficiently [21]).

Table III. - Calculated pure metals properties of $\mathrm{Au}$ and $\mathrm{Ni}$ compared with the experimental values. Elastic constants at $0 \mathrm{~K}$ [43] are in $10^{11} \mathrm{~Pa}$ and the vacancy formation energies in $\mathrm{eV}$ $[44,45]$.

\begin{tabular}{|r|c|c|c|c|}
\hline & $c_{11}$ & $c_{12}$ & $c_{44}$ & $E_{1 \mathrm{v}}^{\mathrm{f}}$ \\
\hline $\mathrm{Au}$ & 2.023 & 1.703 & 0.381 & 0.55 \\
exp. & 2.020 & 1.700 & 0.450 & 0.94 \\
& & & & \\
$\mathrm{Ni}$ & 2.266 & 1.680 & 0.842 & 1.37 \\
exp. & 2.612 & 1.508 & 1.317 & 1.40 \\
\hline
\end{tabular}

The Rose equation of state [10] describes successfully the relation between energy and interatomic distance [22] for a large number of phenomena (bimetallic adhesion, cohesion and chemisorption at a metallic surface). With our notations, the so-called universal equation can be written as :

$$
E(r)=-E_{0}\left[1+\alpha\left(\frac{r}{r_{0}}-1\right)+0.05 \alpha^{3}\left(\frac{r}{r_{0}}-1\right)^{3}\right] \exp \left[-\alpha\left(\frac{r}{r_{0}}-1\right)\right]
$$

with $\alpha=\left(9 B V_{0} / E_{0}\right)^{1 / 2}, V_{0}$. atomic volume.

The values of table I lead to : $\alpha_{\mathrm{Au}}=6.7408$ and $\alpha_{\mathrm{N}_{1}}=5.0873$.

The agreements of the fitted potentials with Rose's curves are verified in figure 1. Reciprocally, the fitted parameters presented in table II can be extracted directly from the fit of equation (1) on Rose's curve for the interval $\left[0.8 r_{0}, 1.4 r_{0}\right]$. The equivalence between the analytic energy-distance relation and the potential derived from the tight-binding model is numerically verified in this case (see the comments in [23]).

These potentials have been checked with LMTO calculations and the agreement is also very good [24]. These checks prove that we obtain a realistic potential at $0 \mathrm{~K}$ for the pure elements, even at distances where the long range anharmonic term becomes important.

2.3 FITTING PROCEDURE FOR ALLOYS. - A classical procedure, used to predict heteroatomic interactions [25, 26] involves fitting the like atom potential interactions to properties of the corresponding pure components (see above) and then averaging the equivalent parameters to obtain the values of the unlike atom interactions. We get a heteroatomic concentrationindependent potential.

This simple method is applied in this paper. We put $r_{0}^{\mathrm{AuNi}}=\left(r_{0}^{\mathrm{AuAu}}+r_{0}^{\mathrm{NiN}}\right) / 2$ using an arithmetic average of $p$ and $q$ and a geometric average of $A$ and $\xi$ (See Tab. IV). 


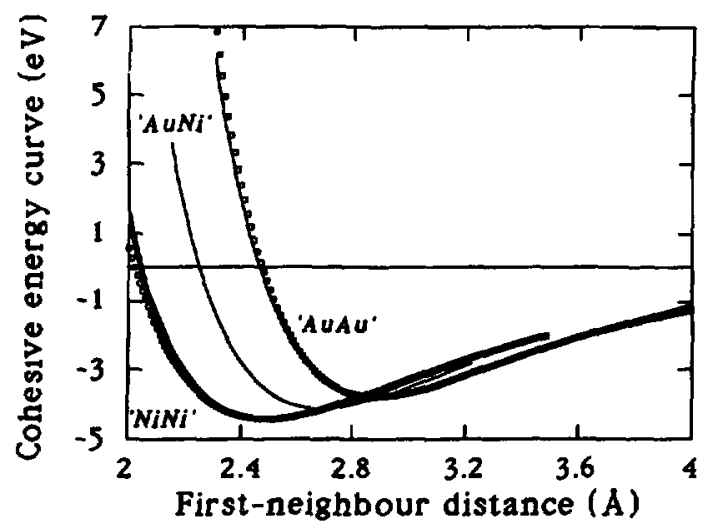

Fig. 1. - The cohesive energy curve versus first-neighbour distance for the pure elements and the crossed term (solid lines). The minimum of the energy for the interspecies interactions is around $4.137 \mathrm{eV}$. Rose's equations of state are drawn with open squares.

Table IV. - Values of the potential parameters for the repulsive and attractive terms of the crossed term. A and $\xi$ are in $\mathrm{eV}, p$ and $q$ are dimensionless and the radii are normalized by $r_{0}=2.6845 \AA$.

\begin{tabular}{|c|c|c|c|c|}
\hline Repulsive term & $A$ & $p$ & $r_{\mathrm{t}}$ & $r_{\mathrm{c}}$ \\
\hline «AuNi » & 0.1855 & 10.0456 & 1.2 & 1.5336 \\
\hline
\end{tabular}

\begin{tabular}{|c|c|c|c|c|}
\hline Attractive term & $\xi$ & $q$ & $r_{\mathrm{t}}$ & $r_{\mathrm{c}}$ \\
\hline «AuNi » & 1.8182 & 3.5049 & 1.2 & 1.6780 \\
\hline
\end{tabular}

This approximation may be partially justified [27] for the attractive part of transition metal alloys without diagonal disorder [28] (when the atomic energy levels are nearly the same). The dependence of the hopping integrals on the atoms at different sites is only considered as the offdiagonal disorder.

We plot in figure 1 the cohesive energy curve of a monoatomic hypothetical fcc system which would have this average interaction, thus allowing to compare the heteroatomic potential to those of pure metals.

\section{Alloying energies.}

The energy of formation (i.e. the enthalpy of formation at zero pressure) of $\mathrm{Au}_{1-x_{x}} \mathrm{Ni}_{x}$ solid solution is defined by :

$$
E_{\text {form. }}(x)=E(x)-(1-x) E_{0}^{\mathrm{Au}}-x E_{0}^{\mathrm{N} 1}
$$

where $E(x)$ is the total energy per atom of a chemically random crystal. We obtain $E(x)$ by a numerical relaxation of atomic positions of an initial system to reach a (local) 
minimum. This initial configuration consists of a large cell with $N=4000$ sites extended over the whole space by periodic boundary conditions. The cell is defined by a fcc alloy at a given concentration with the lattice parameter of Vegard's law (linear interpolation between the lattice parameters of the pure metals). We randomly choose the chemical nature of the fcc sites, because the first Warren-Cowley short range parameters are nearly zero $[6,7]$ as has been shown experimentally.

By using a Monte Carlo technique, we have built a configuration which has the experimental Warren-Cowley parameters at the concentration $x=0.4$ where the short range order is known [6]. Its formation energy has been calculated to be only $3 \%$ lower than that of the completely random configuration. This small value has justified the use of completely random alloys in this study.

The sensitivity towards the number of atoms has been checked and no significant deviation has been measured.

The reduced co-ordinates (in a unit-length box) and the faces of the orthorhombic cell are free to move independently during the relaxation at zero pressure (for the constant volume relaxation used in Sect. V, the box sides are fixed). The minimization of the energy is achieved numerically using the conjugate gradient method until $\Delta E<10^{-10} \mathrm{eV}$. So, the local relaxations are fully treated (without the approximations of [26]).

The energy of formation (at zero pressure and zero temperature) versus concentration is presented in figure 2 . The energy curve is very close to the estimated experimental curve at $0 \mathrm{~K}$ deduced from the measurements of Hultgren at $1150 \mathrm{~K}$ [9]. This extrapolation at $0 \mathrm{~K}$ is done with the Kirchoff relation and the $C_{p}$ measurements :

$$
\Delta H_{\mathrm{T}}=\Delta H_{\mathrm{T}_{0}}+\int_{T_{0}}^{T} \Delta C_{p} \mathrm{~d} T .
$$

The sign of the formation energy agrees with the phase diagram: the simulated alloy is metastable at zero temperature and is obtained, experimentally, by quenching.

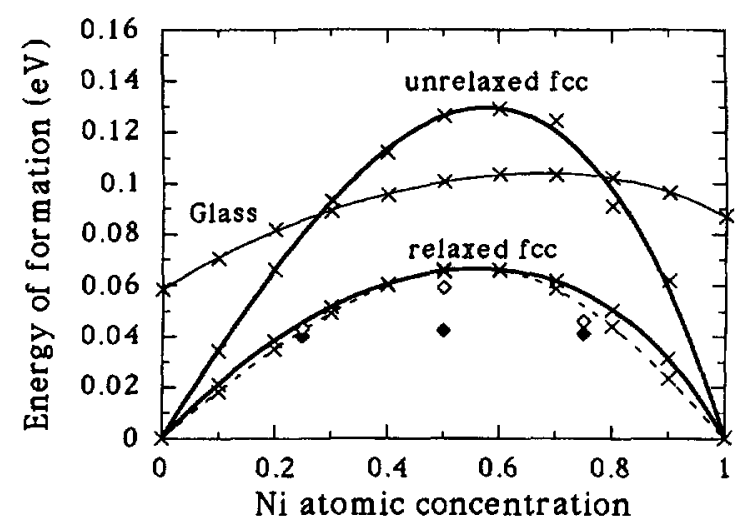

Fig. 2. - Formation energies of the $\mathrm{Au}_{1-}{ }_{x} \mathrm{Ni}_{x}$ solid solutions and glasses versus $\mathrm{Ni}$ concentration. The crosses $(x)$ indicate the results obtained in this work (with 4000 atoms): the unrelaxed energy is calculated with Vegard's law lattice parameter, this is the initial state for the relaxation at zero pressure. The dotted line extrapolates at $0 \mathrm{~K}$ the experimental results of Hultgren (see text). Three computed metastable compounds are drawn on this figure (white rhombus : initial state; black rhombus : relaxed state). 
The decrease of energy between initial and relaxed configurations is very large in this system. This shows the importance of accurately treating the local relaxation effect, otherwise the energy could be overestimated by a factor of two.

The alloy heats of solution for single substitutional impurities (1 over 4000$)$ are computed with a zero-pressure relaxation. These quantities allow a test of our interactions whereas they are input parameters for EAM potentials [15, 16]. The available thermodynamic data [9] concern the high temperature range $(1150 \mathrm{~K})$ and no attempt has been made to extrapolate those values to $0 \mathrm{~K}$. For the $\mathrm{Au}$ in $\mathrm{Ni}$, the calculated (resp. measured) value is $0.389 \mathrm{eV}$ (resp. $0.285 \mathrm{eV}$ ) and for the $\mathrm{Ni}$ in $\mathrm{Au}: 0.227 \mathrm{eV}$ (resp. $0.221 \mathrm{eV}$ ).

To show that the segregation behaviour is induced by the frustration effect, we also evaluated the chemical driving force without this lattice frustration.

The interaction potential (1) depends on the reduced variable $r_{i j} / r_{0}^{\mathrm{AB}}$ where $\mathrm{A}$ and B (Au or $\mathrm{Ni}$ ) are the types of atoms $i$ and $j$ respectively. To calculate the energy of a hypothetical fcc unfrustrated lattice with a given chemical configuration, we compute the energy using equation (1). The parameters of tables II and IV depend on each pair type but are normalized for each distance $r_{i j}$ by the same arbitrary unit length $r_{0}$. This can be considered as the energy of a lattice with distance distortions that cancel the frustration effects. Of course such a lattice cannot exist in a 3D Euclidien space but this trick removes the « size effect » resulting from the potential. The energy of formation is computed from equation (3) for the whole concentration range (a small relaxation is still present since the environment of each atom is generally not chemically centrosymmetric). Now, this energy of formation exhibits a negative sign, demonstrating an heterocoordination tendency (the ordering energy computed with potential depths of figure 1 is in agreement with this behaviour). Thus the segregation tendency in the $\mathrm{Au}_{1-{ }_{x}} \mathrm{Ni}_{x}$ solid solutions is due to size effect frustration (« elastic effect ») exceeding the heterocoordination tendency ( chemical effect").

We have also built $\mathrm{Au}_{1-x} \mathrm{Ni}_{x}$ amorphous structures using numerical relaxation. The starting configurations are dense random sets of 4000 positions with exclusion distances $r_{0}^{\mathrm{AB}}$ and with $\mathrm{Ni}$ concentration $x$. Figure 2 shows the energy versus concentration. The glass structure is less stable than the fcc solid solution. However, since there is less frustration in the amorphous state than in the fcc lattice, the formation energy of the glass structure (referred to the pure glass constituents) is twice as small as that of the crystalline structure.

Finally, the metastable compounds $\mathrm{AuNi}\left(\mathrm{Ll}_{0}\right)$ and $\mathrm{Au}_{3} \mathrm{Ni}, \mathrm{AuNi}_{3}\left(\mathrm{Ll}_{2}\right)$ are computed, and depicted in figure 2. The second moment approximation is in principle not sufficient to account properly for chemical ordering effects [28], and this potential certainly not. But it is reasonable to get compounds which are slightly more stable than the solid solutions. Some authors suggest their formations [29] from successive annealings at various temperatures.

\section{Topological properties.}

The $\mathrm{Au}_{1-x} \mathrm{Ni}_{x}$ system is, to our knowledge, the only fec metallic alloy with EXAFS data available for the whole composition range [7]. The lattice parameter $a$ is measured from X-ray diffraction and, in the simulation, is computed from the size of the periodic box which gives an averaged first neighbour distance $a / \sqrt{2}$. We can also define an average lattice and, figure 3 shows the corresponding displacement distribution for an equiconcentration alloy.

Figure 4 shows that the positive deviation of the first-neighbour distance from Vegard's law is well reproduced. This deviation has been predicted by a mean field theory [30] and its extensions [31]. The infinite dilution limit leading to the strong asymmetry of the curve has also been reproduced quantitatively: when $x \rightarrow 0$ (resp. $x \rightarrow 1$ ), the simulation predicts $\mathrm{d} a / \mathrm{d} x=-0.42$ (resp. $\mathrm{d} a / \mathrm{d} x=-0.79$ ) and the experiment -0.4 [7] (resp. -0.8 ). 


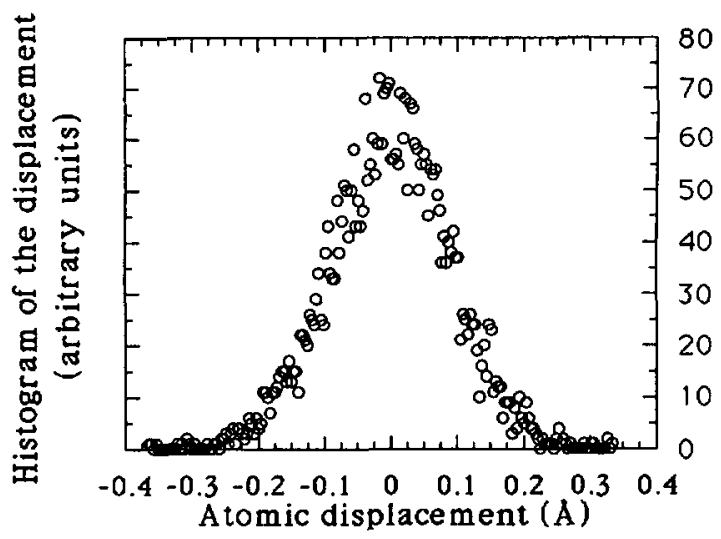

Fig. 3. - Histogram of the atomic position deviation from the initial unrelaxed state for $x=0.5$ along the [100] direction of the cubic cell (the results are similar for the [010] and [001] directions).

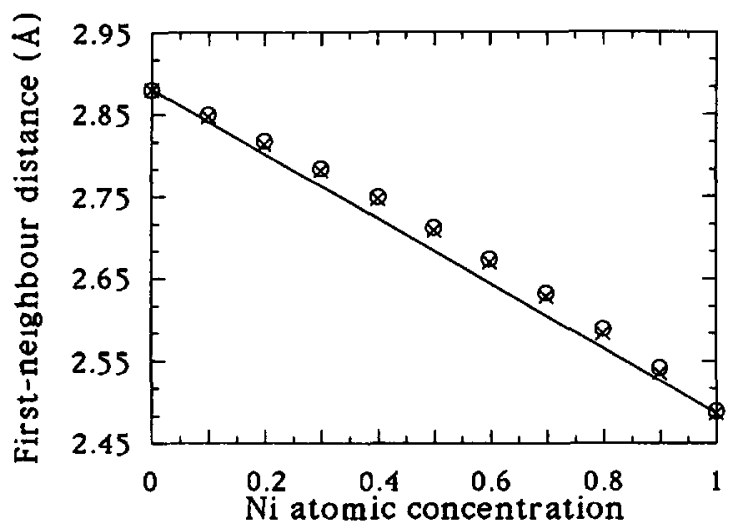

Fig. 4. - Deviation from Vegard's law of the $\mathrm{Au}_{1-} \mathrm{Ni}_{x}$ solid solutions versus $\mathrm{Ni}$ concentration. Open circles represent numerical simulation and crosses the experimental results of Renaud [7].

The reduced partial pair correlation function $g_{\mathrm{AB}}(r)$ is related to the radial distribution function $\mathrm{d} N / \mathrm{d} r$ ( $N$ is the average number of $\mathrm{B}$-atoms contained between the two spheres of radius $r$ and $r+\mathrm{d} r$ and centered on an A-atom):

$$
\frac{\mathrm{d} N}{\mathrm{~d} r}=4 \pi \rho_{\mathrm{B}} r^{2} g_{\mathrm{AB}}(r)
$$

$\mathrm{A}$ and $\mathrm{B}$ are $\mathrm{Au}$ or $\mathrm{Ni}$ elements, $\rho_{\mathrm{B}}$ is the atomic density of the $\mathrm{B}$ atoms.

In spite of the large displacements of the atoms, the peaks of the partial pair correlation functions correspond to those of the fcc lattice. The first neighbour co-ordination shells can be isolated clearly from the second ones for the whole concentration range (the same applies to larger distances).

The partial pair correlation functions restricted to the first neighbour shell are shown in figure 5 for equiconcentration. A lot of information is contained in these curves.

First of all, we deduce the partial averaged first-neighbour distances $\bar{R}_{\mathrm{AB}}$ plotted in figure 6 


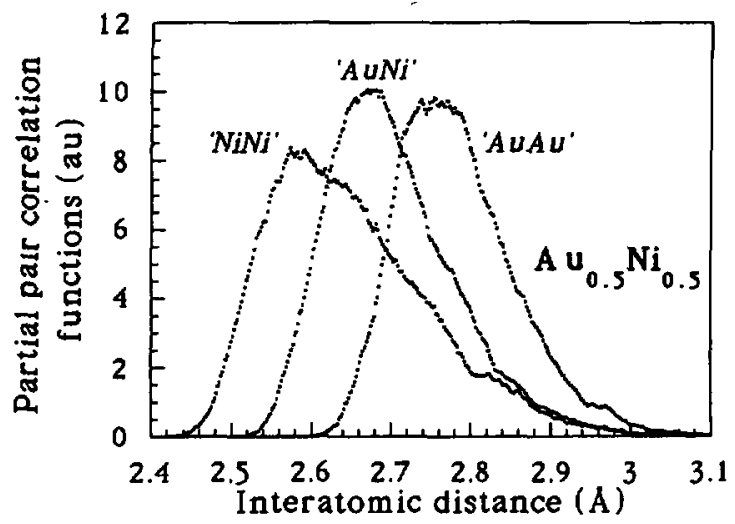

Fig. 5. - The three partial pair correlation functions $g_{i j}(r)$ for $x=0.5$ obtained after zero pressure relaxation.

- NiNi calc. $\square$ AuNi calc. - AuAu calc.

- NiNi exp. - AuNi exp. - AuAu exp.

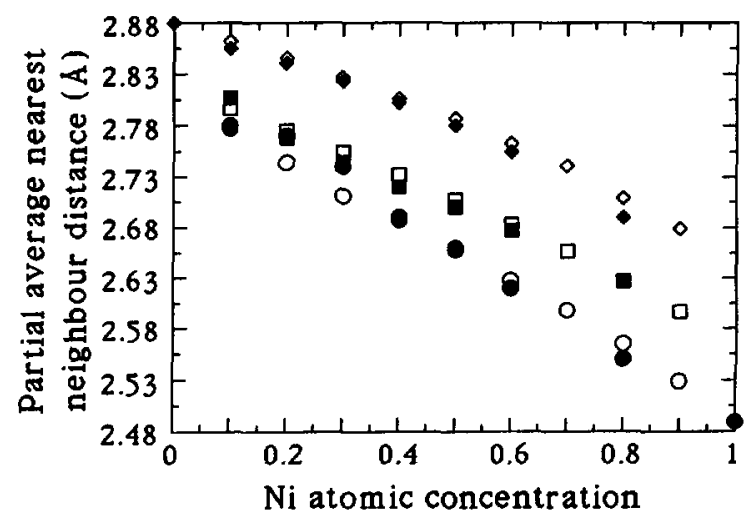

Fig. 6. - The mean $\mathrm{Au}-\mathrm{Au}$ (rhombs), $\mathrm{Au}-\mathrm{Ni}$ (squares) and $\mathrm{Ni}-\mathrm{Ni}$ (circles) nearest neighbour distance in the $\mathrm{Au}_{1-x} \mathrm{Ni}_{x}$ solid solutions versus $\mathrm{Ni}$ concentration. White symbols represent simulation and black symbols experimental data [7].

$\left(\bar{R}_{\mathrm{AB}}=\int r^{3} g_{\mathrm{AB}}(r) \mathrm{d} r / \int r^{2} g_{\mathrm{AB}}(r) \mathrm{d} r\right.$ where the integration is done over the first peak). The agreement between simulation and EXAFS experiments [7] is noteworthy. Then, we plot the second and third moment of these distributions $\left(\sigma_{i}=\left\langle\left(r-\bar{R}_{\mathrm{AB}}\right)^{\imath}\right\rangle, i=2,3\right)$ in figure 7 . These results which measure the widths and asymmetries respectively are also in quantitative agreement with the measurements of Renaud et al. [7]. For the equiconcentration, the experimental measurements are : $\sigma_{2}^{\mathrm{NiNi}} \simeq 13 \times 10^{-3} \AA^{2}, \quad \sigma_{2}^{\mathrm{AuN} N_{1}} \simeq 7 \times 10^{-3} \AA^{2}, \quad \sigma_{2}^{\mathrm{AuAu}} \simeq$ $3 \times 10^{-3} \AA^{2}, \quad \sigma_{3}^{\mathrm{NiNi}} \simeq 11 \times 10^{-4} \AA^{3}, \quad \sigma_{3}^{\mathrm{AuN} 1} \simeq 5 \times 10^{-4} \AA^{3}, \quad \sigma_{3}^{\mathrm{AuAu}} \simeq 2 \times 10^{-4} \AA^{3}$ and these values are close to the simulated ones.

In agreement with the results of Renaud et al. [7] and $\mathrm{Wu}$ and Cohen [6], the width and the asymmetry of $\mathrm{Ni}-\mathrm{Ni}$ pair distribution functions are larger than $\mathrm{Au}-\mathrm{Ni}$ ones which are also larger 

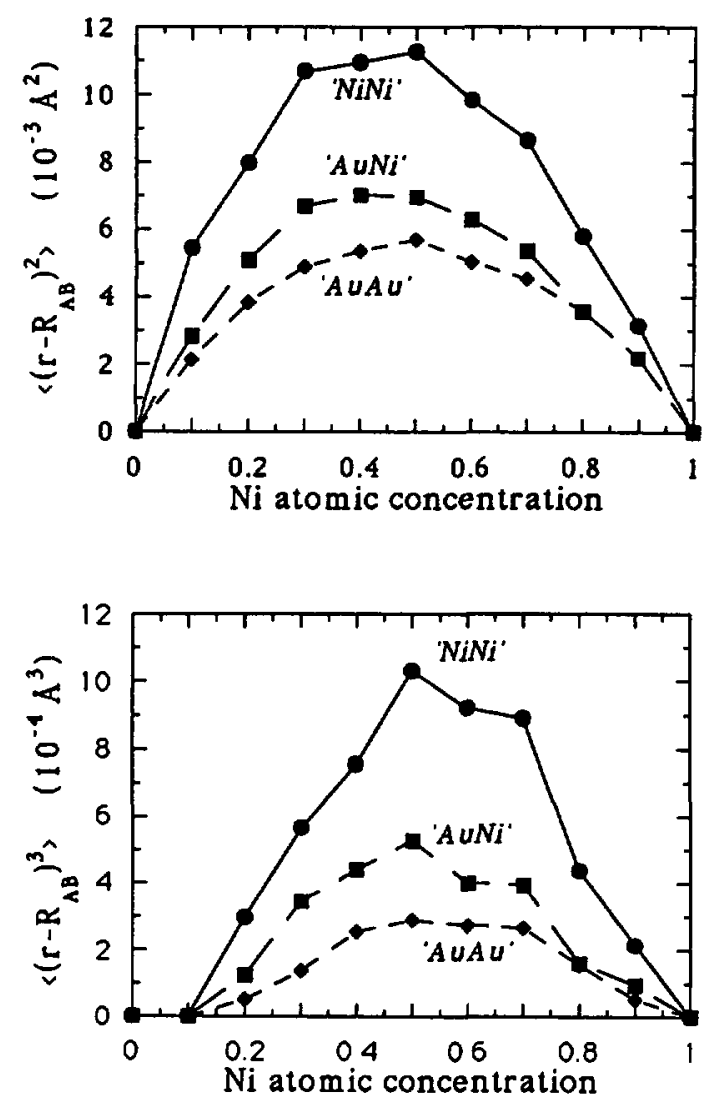

Fig. 7. - Second and third moment of the partial pair correlation functions in the $\mathrm{Au}_{1-{ }_{x}} \mathrm{Ni}_{x}$ solid solutions model versus $\mathrm{Ni}$ concentration showing evolutions of the widths and asymmetries of the three pair correlation functions.

than $\mathrm{Au}-\mathrm{Au}$ ones. Moreover $\bar{R}_{\mathrm{Au}-\mathrm{N} 1}$ is always closer to $\bar{R}_{\mathrm{Ni}-\mathrm{Ni}}$ than to $\bar{R}_{\mathrm{Au}-\mathrm{Au}}$. These trends of distance distributions can be understood by considering that the $\mathrm{Ni}$ atoms can adjust to the topological frustration more easily than gold atoms, because the energy cost is higher for contraction than for dilation due to the anharmonicity of the interatomic interactions (see Fig. 1).

A new description can be developed from the study of interstitial sites. The $3 \mathrm{~N}$ interstitial sites ( $2 N$ tetrahedral and $N$ octahedral, if $N$ is the atom number) in the perfect lattice are exactly known. For the distorted structures, two types of analyses are performed : first, the Voronoï polyhedra [32] which consider only positions of atomic sites; and secondly, radical planes [33] where size effect may be introduced. The bond lengths of figure 6 are an excellent example. These constructions define partitions of the whole space and tetrahedron networks are associated with them (Delaunay's network in the case of the Voronoï construction). These tetrahedron networks also form partitions of space and allow the definition of the interstitial sites [34].

These two different approaches lead to the same conclusions :

(i) The initial tetrahedral sites are still present in the relaxed state (the two lists of atoms defining the relaxed and unrelaxed tetrahedral sites are identical). 
(ii) Each initial octahedral site is transformed into a distorted octahedron which can be decomposed into 4 tetrahedra or in few cases into 5 tetrahedra.

The histograms of the volumes of the tetrahedral sites (see Fig. 8) can be decomposed into partial distributions of the volumes $V_{\mathrm{Au}_{4-1} \mathrm{~N}_{1_{1}}}(i=0 . .4)$ corresponding to the five chemical arrangements : $\mathrm{Au}_{4}, \mathrm{Au}_{3} \mathrm{Ni}, \mathrm{Au}_{2} \mathrm{Ni}_{2}, \mathrm{AuNi}_{3}, \mathrm{Ni}_{4}$ (the octahedral analysis can be performed with the same method, however it is more complex because of the larger number of possible combinations). The average positions of these five peaks are plotted in figure 9. They are distinct from the simple predictions of the tetrahedral volumes :

$$
V_{\mathrm{Au}_{4}}^{0}=\frac{a^{3}}{6 \sqrt{2}}, \quad V_{\mathrm{Au}_{3} \mathrm{Ni}_{1}}^{0}=\frac{a^{2}}{4} \sqrt{c^{2} / 3-a^{2} / 9}, \quad V_{\mathrm{Au}_{2} \mathrm{~N}_{2}}^{0}=\frac{a b}{6} \sqrt{c^{2}-\left(a^{2}+b^{2}\right) / 4}
$$

based on the partial first-neighbour distances: $a=\bar{R}_{\mathrm{AA}}, b=\bar{R}_{\mathrm{BB}}$ and $c=\bar{R}_{\mathrm{AB}}$ (the other volumes are obtained by permutation).

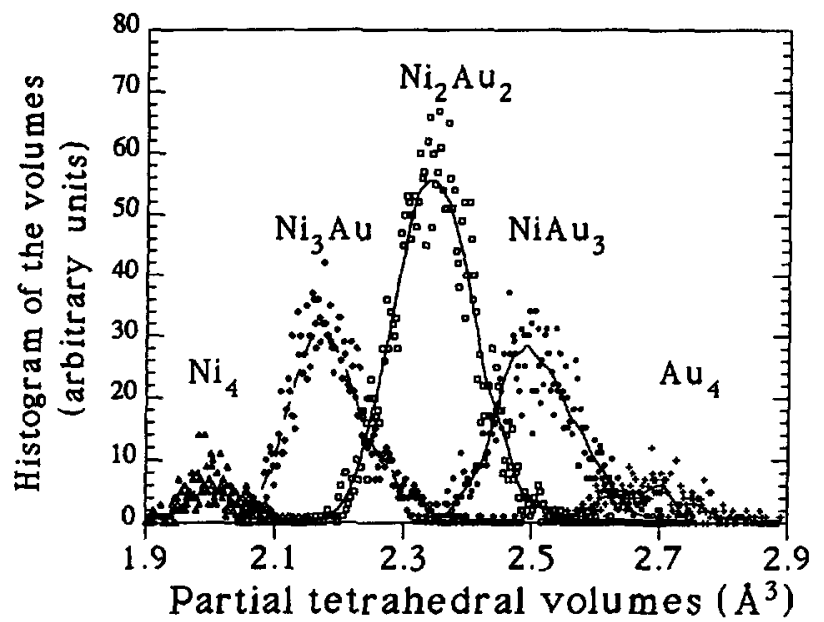

Fig. 8. - Histogram of the tetrahedral volumes for $x=0.5$ decomposed in the partial volumes of the tetrahedra: $\mathrm{Au}_{4}, \mathrm{Au}_{3} \mathrm{Ni}, \mathrm{Au}_{2} \mathrm{Ni}_{2}, \mathrm{AuNi}_{3}$ and $\mathrm{Ni}_{4}$. The lines are weighted smooths of the data.

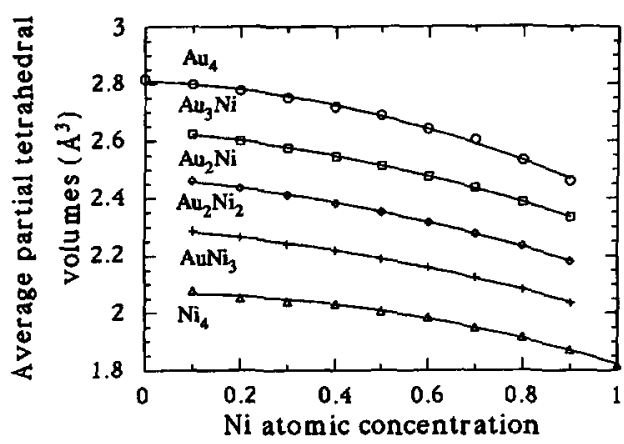

Fig. 9. - Averages volumes of each type of the tetrahedral sites in the $\mathrm{Au}_{1-}{ }_{-} \mathrm{Ni}_{x}$ solid solutions versus $\mathrm{Ni}$ concentration. 
This discrepancy results from the correlation between the length of the atomic pairs and the tetrahedron types (this correlation is weaker in the amorphous structure [34]).

The analytical expressions give the correct volumes only when the matrix effect is low i.e. when the considered tetrahedron is in a majority : near $x=0(x=1)$ for $V_{\mathrm{Au}_{4}}^{0}\left(V_{\mathrm{Ni}_{4}}^{0}\right)$, near $x=1 / 4(x=3 / 4)$ for $V_{\mathrm{Au}_{3} \mathrm{Ni}^{0}}^{0}\left(V_{\mathrm{AuNi}_{3}}^{0}\right)$ and near $x=1 / 2$ for $V_{\mathrm{Au}_{2} \mathrm{Ni}_{2}}^{0}$.

It is noteworthy that the behaviour of the interstitial volumes described in this text affects the diffusion properties of solid solutions.

\section{Elastic behaviour.}

5. 1 Computational METHOD. - At zero temperature, three main numerical methods can be used to evaluate the elastic constants of an ideal crystal (having one or several atoms per unit cell) or inhomogeneous systems (for example grain-boundary superlattices [35]) : (i) the local elastic-constants tensor [36] (ii) the lattice-dynamics [36] (iii) the finite-strain method. This third method is applied to the large systems in this paper.

The equilibrium states are relaxed at zero pressure; $E_{0}$ is the energy and $V_{0}$ is the volume per atom. We apply small strains by changing the shape or the volume of the periodic cell which contains the $N$ atoms and afterwards we relax the system, keeping this new cell fixed. These strains may be defined with only one distortion variable $\varepsilon$ by a matrix $J(\varepsilon)$ transforming the box (and lattice) vectors $[37,38]$. This matrix defines the Lagrangian strain $\eta(\varepsilon)=$ $\left(J^{t} J-I\right) / 2$ where $I$ is the identity matrix.

The cubic macroscopic systems only have three second order independent elastic constants.

Using the macroscopic deformation matrix :

$$
J(\varepsilon)=S(\varepsilon)\left(\begin{array}{ccc}
1+\delta_{1} \varepsilon & \delta_{6} \varepsilon & \delta_{5} \varepsilon \\
\delta_{6} \varepsilon & 1+\delta_{2} \varepsilon & \delta_{4} \varepsilon \\
\delta_{5} \varepsilon & \delta_{4} \varepsilon & 1+\delta_{3} \varepsilon
\end{array}\right)
$$

we can get $\Delta E / V_{0}$ in terms of $J(\varepsilon)$.

The shear moduli $c^{\prime}=\left(c_{11}-c_{12}\right) / 2$ and $c_{44}$ can be extracted with the strains defined by parameters $\delta_{1}$ and the normalization function $S(\varepsilon)$ given in table $\mathrm{V}\left({ }^{*}\right)$. Strain number 1

Table V. - Strains to isolate elastic constants (see text for notations). Strains number 1 and 2 are volume-conserving and strains number 3 and 4 are not.

\begin{tabular}{|c|c|c|c|c|c|c|c|c|}
\hline Strain & $\delta_{1}$ & $\delta_{2}$ & $\delta_{3}$ & $\delta_{4}$ & $\delta_{5}$ & $\delta_{6}$ & $S(\varepsilon)$ & $\Delta E /\left(V_{0} \varepsilon^{2}\right)$ \\
\hline 1 & 1 & 0 & 0 & 0 & 0 & 0 & $(1+\varepsilon)^{-1 / 3}$ & $\frac{2}{3}\left(c_{11}-c_{12}\right) / 2$ \\
2 & 0 & 0 & 0 & 1 & 1 & 1 & $\left(1-3 \varepsilon^{2}+2 \varepsilon^{3}\right)^{-1 / 3}$ & $\frac{3}{2} c_{44}$ \\
3 & 1 & 0 & 0 & 0 & 0 & 0 & 1 & $\frac{1}{2} c_{11}$ \\
4 & 1 & 1 & 1 & 0 & 0 & 0 & 1 & $\frac{9}{2} B$ \\
\hline
\end{tabular}

$\left(^{*}\right)$ The lower-case letter indicates that elastic constants are computed in a non-primitive set of axes of the fcc structure, i.e. the cubic axes: ([100], [010], [001]). 
(related to $c^{\prime}$ ) only involves tensile strain along the cubic axes. Strain number 2 (related to $c_{44}$ ) leads to a distortion of the orthorhombic box.

It is more convenient to choose an initial cell constructed in the set of perpendicular axes : $\left(\frac{1}{\sqrt{2}}[1 \overline{1} 0], \frac{1}{\sqrt{6}}[11 \overline{2}], \frac{1}{\sqrt{3}}[111]\right)$ and to apply the strain :

$$
J(\varepsilon)=\left(1-3 \varepsilon^{2}-2 \varepsilon^{3}\right)^{-1 / 3}\left(\begin{array}{ccc}
1+\varepsilon & 0 & 0 \\
0 & 1+\varepsilon & 0 \\
0 & 0 & 1-2 \varepsilon
\end{array}\right)
$$

Another macroscopic deformation is necessary to separate $c_{11}$ and $c_{12}$ from $c^{\prime}$. It may be done with the strains 3 or 4 (see Tab. V). Strain 3 allows us to obtain directly $c_{11}$ and strain 4 yields the bulk modulus $B$ (cubic lattices satisfy the relation $B=\left(c_{11}+2 c_{12}\right) / 3$ ).

The magnitude of $\varepsilon$ chosen to compute $c_{I J}$ must be large enough to neglect residual numerical errors and small enough to neglect non-linear effects due to higher elastic contributions. The second order elastic constants are deduced from the energy-deformation curves $E(\varepsilon)$. The anharmonic term leads to a first error bar for the determination of elastic constants; this contribution is quite easy to extract with the curves $E(\varepsilon)$. A second one comes from the statistical error made by choosing an initial chemically random configuration. To estimate these errors, we have computed the elastic constant $c^{\prime}$ and the bulk modulus $B$ at $x=0.5$ for five initial random configurations and five deformations ranging from $0.05 \%$ to $0.5 \%$ (a classical value being around $0.1 \%$ ). A maximum margin of error of $2 \%$ on the elastic constants has been found and the error bars are reported in the figures 10, 11 and 12.

5.2 RESULTS. - The elastic constants shown in figures 10 and 11 may be compared to the experimental results of Golding et al. [5] (measured at room temperature for $x \leqslant 0.42$ ) and Renaud et al. [7] (at room temperature for $x=0.20$ ). The samples do not have the same metallurgical treatment: Golding's samples are directly quenched from the complete miscibility range and Renaud's samples exhibit the short range order of the miscibility gap after an ageing at $520 \mathrm{~K}$. For these particular concentrations, the elastic constants always have the same signs of deviation from the linear interpolations which are in agreement with our results : $c_{11}, c_{44}$ and $c^{\prime}$ are softened by the solid solution effect whereas $c_{12}$ is enhanced. The simulation predicts furthermore that the maximum is around $x=0.65$ (see Fig. 12). The

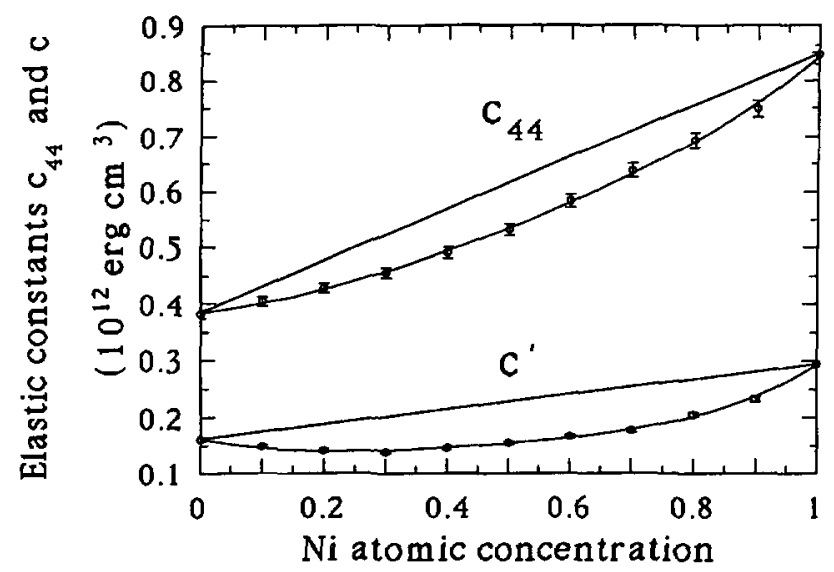

Fig. 10. - Elastic constants $c_{44}$ and $c^{\prime}$ in $10^{11} \mathrm{~Pa}$ calculated with volume-conserving strains number 1 and 2 of table V. See text for error bars. The lines are only a guide for the eyes. 


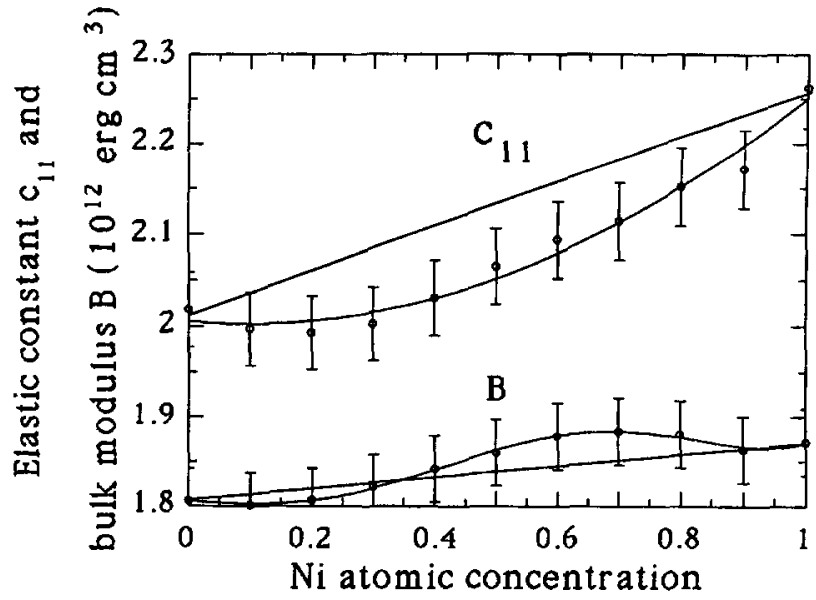

Fig. 11. - Elastic constant $c_{11}$ and bulk modulus $B$ in $10^{11} \mathrm{~Pa}$ calculated with volume-non-conserving strains 3 and 4 of table V. See text for error bars.

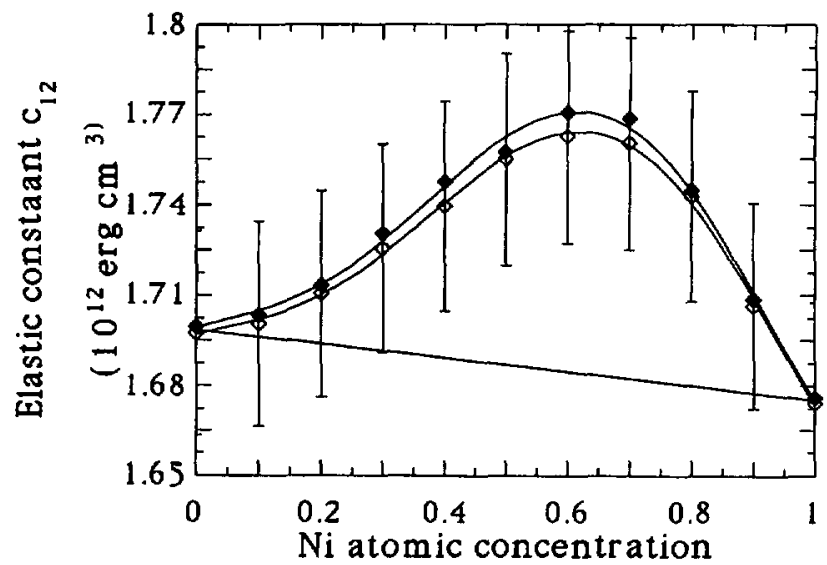

Fig. 12. - Elastic constant $c_{12}$ in $10^{11} \mathrm{~Pa}$ calculated from the relation $c_{12}=\left(3 B-c_{11}\right) / 2$ (dark rhombs) and from $c_{12}=c_{11}-2 c^{\prime}$ (open rhombs) with the data of figures 9 and 10 . See text for error bars.

quantitative agreement between the calculated and experimental elastic constants corresponds to the one obtained above for the pure crystals. This is only a rough comparison since the experiments are not at $0 \mathrm{~K}$. For instance at $x \simeq 0.2$, we get :

$$
\begin{array}{lllll}
c_{11}^{\text {calc. }}=1.99, & c_{11}^{[5]}=1.96 & \text { and } & c_{11}^{[7]}=1.95 & \left(10^{11} \mathrm{~Pa}\right) \\
c_{12}^{\text {calc. }}=1.71, & c_{12}^{[5]}=1.58 & \text { and } & c_{12}^{[7]}=1.68 & \left(10^{11} \mathrm{~Pa}\right) \\
c_{44}^{\text {calc. }}=0.43, & c_{44}^{[5]}=0.51 & \text { and } & c_{11}^{[7]}=0.42 & \left(10^{11} \mathrm{~Pa}\right) .
\end{array}
$$

The behaviour of the bulk modulus $B$ is more complex : starting with a softening, it is followed by an enhancement, but this effect is small. The Debye temperature $\theta_{\mathrm{D}}$ can be calculated [39] from the elastic constants and the vibrating free energy can be deduced. This allows one to compute the miscibility gap [40] of the system. 


\section{Conclusion.}

This paper deals with the alloying, topological, and elastic properties of the $\mathrm{Au}_{x} \mathrm{Ni}_{1-x}$ solid solutions at $0 \mathrm{~K}$ studied by numerical relaxation.

The empirical interatomic potential is based on the second moment approximation of the tight binding theory of metallic cohesion. With a very simple average rule for interspecies interactions, it is shown that the local relaxation is of paramount importance on the alloying energy because of the large difference between atomic sizes of individual species.

The available experimental results are checked by the potential and the evolution of the properties are extended over the whole concentration range.

The formation energy of solid solutions agrees with the extrapolation at $0 \mathrm{~K}$ of the experimental measurements. The relative phase stability of glass and compounds is studied with the same method.

The bond length distributions compare well with EXAFS experiments and Johnson embedded-atom potential [41]. It is confirmed that the bonding frustration by the lattice plays an important role (previously called the elastic effect).

Moreover, a new study of interstitial sites shows that the chemical effect induces a splitting of the tetrahedral volumes. This allows a characterization of the structure and may have implications for the interstitial diffusion.

Finally, we analyzed the influence of the topological disorder on the elastic constants. This effect is found to be low.

\section{Acknowledgments.}

We would like to thank A. Pasturel for LMTO calculations and also G. M. Moreau and G. Lechevallier for useful computer programs.

\section{References}

[1] Lu Z. W., Wei S.-H., Zunger A., Phys. Rev. B 45 (1992) 10314.

[2] Bose S. K., Kurdrnovsky J., Jepsen O. and Andersen O. K., Phys. Rev. B 45 (1992) 8272.

[3] Lu Z. W., Wei S.-H., Zunger A., Frota-Pessoa S. and Ferreira L. G., Phys. Rev. B 44 (1991) 512.

[4] Bozzolo G., Ferrante J. and Smith J. R., Phys. Rev. B 45 (1992) 493.

[5] Golding B., Moss S. C. and Averbach B. L., Phys. Rev. 148 (1967) 637.

[6] Wu T. B., COHEN J. B. and Yelon W., Acta metall. 30 (1982) 2065.

Wu T. B. and COHEN J. B., Acta metall. 31 (1983) 1929.

WU T. B. and COHEN J. B., Acta metall. 32 (1984) 861.

[7] Renaud G., Motta N., Belakhovsky M., Lançon F. and Billard L., Solid State Commun. 63 (1987) 569.

Renaud G., Motta N., Lançon F. and Belakhovsky M., Phys. Rev. B 38 (1988) 5944.

Renaud G., PhD thesis, Université Joseph Fourier, Grenoble I (1988).

[8] Hersant D., PhD thesis, Université de Paris Sud, Orsay (1991).

[9] Hultgren R., ORR R. L., Andersen P. D. and Kelley K. K., Selected Values of the Thermodynamic Properties of Binary Alloys (Wiley, New York, 1963).

[10] Rose J. H., Smith J. R., Guinea F. and Ferrante J., Phys. Rev. B 29 (1984) 2963.

[11] Ducastelle F., J. Phys. France 31 (1970) 1055.

[12] Cyrot-Lackmann F., J. Phys. Chem. Solids 29 (1968) 1235.

CYrot-LACKMANN F., Surface Sci. 15 (1969) 535. 
[13] Guillopé M. and Legrand B., Surface Sci. 215 (1989) 577.

[14] Rosato V., Guillopé M. and Legrand B., Philos. Mag. A 59 (1989) 321.

[15] Daw M. S. and Baskes M. I., Phys. Rev. B 29 (1984) 6443.

[16] Folles S. M., Baskes M. I. and Daw M. S., Phys. Rev. B 33 (1986) 7983.

[17] Finnis M. W. and Sinclair J. E., Philos. Mag. A 50 (1984) 45.

[18] Sutton A. P. and Chen J., Philos. Mag. Lett. 61 (1990) 139.

[19] EYMERY J., PhD Thesis, INPG Grenoble (1992).

[20] Martin J. W., J. Phys. C : Solid State Phys. 8 (1975) 2837 and 2858.

[21] Masuda K., Hamada N. and Terakura K., J. Phys. F : Met. Phys. 14 (1984) 47.

[22] BanerJea A. and Smith R., Phys. Rev. B 37 (1988) 6632.

[23] Spanjaard D. and Desjonquères M. C., Phys. Rev. B 30 (1984) 4822.

[24] EYMERY J. and PASTURel A., submitted to publication.

[25] Rafir H. and Sutton A. P., Phil. Mag. Lett. 63 (1991) 217.

[26] Ackland G. J. and Vitek V., Phys. Rev. B 41 (1990) 10324.

[27] Shiba H., Progr. Theo. Phys. 46 (1971) 77.

[28] Ducastelle F., in Computer simulation in Materials Science, M. Meyer and V. Pontikis Eds., NATO ASI Series (Kluwer Academic Publishers, 1991).

[29] Sanadze V. V. and Gulyaev G. V., Sov. Phys. Crystallogr. 4 (1960) 496 and 646.

Khan I. H. and Francombe M. H., J. Appl. Phys. 36 (1965) 1699.

Tsaur B. Y. and MaEnPaA M., J. Appl. Phys. 52 (1981) 728.

[30] Friedel J., Adv. Phys. 3 (1954) 446.

[31] Froyen S. and Herring C., J. Appl. Phys. 52 (1981) 7165.

[32] Frost H. J., Acta metall. 30 (1982) 889.

[33] Gellatly B. J. and Finney J. L., J. Non-Cryst. Solids 50 (1982) 313.

[34] Lançon F., Billard L. and Chamberod A., J. Phys. France 46 (1985) 243.

[35] Kluge M. D., Wolf D., Lutsko J. F. and Phillpot S. R., J. Appl. Phys. 67 (1990) 2370.

[36] Lutsko J. F., J. Appl. Phys. 64 (1988) 1152.

Lutsko J. F., J. Appl. Phys. 65 (1989) 2991.

[37] Cousins C. S. G., Proc. Phys. Soc. 91 (1967) 235.

[38] Nye J. F., Physical Properties of Crystals. Their representation by Tensors and Matrices, Oxford University Press (1957). Reprinted 1989.

[39] Moruzzi V. L., Janak J. F. and Schwarz K., Phys. Rev. B 37 (1988) 790.

[40] PASTUREL A. and EYMERY J., submitted to publication.

[41] Mousseau N. and ThORpe M. F., Phys. Rev. B 45 (1992) 2015.

[42] Kittel C., Introduction à la Physique de l'Etat Solide, Paris Dunod (1972).

[43] SImmons G. and WANG H., Single Crystal Elastic Constants and Calculated Properties : A Handbook, The M.I.T. Press (1971), Second Edition.

[44] Siegel R. W., J. Nucl. Mat. 69 \& 70 (1978) 117.

[45] Seeger A., Schumacher D., SChilling W. and Diehl J., Vacancies and Interstitials in Metals, North Holland, Amsterdam (1970). 